\title{
A Nonlinear Adaptive Predictive Control Algorithm Based on OFS Model
}

\author{
Haitao Zhang 1,2, Zonghai $\mathrm{Chen}^{2}$, Ming $\mathrm{Li}^{2}$, Wei Xiang ${ }^{2}$, and Ting Qin ${ }^{2}$ \\ ${ }^{1}$ Department of Control Science and Engineering, Huazhong University of Science and \\ Technolog, Wuhan, 430074, P.R.China \\ zht@mail.ustc.edu.cn \\ ${ }^{2}$ Department of Automation, University of Science and Technology of China, \\ Hefei, 230027, P.R.China
}

\begin{abstract}
Firstly, a method is introduced which uses Volterra series deploying technique to construct a nonlinear model based on OFS model. Then an improved novel incremental mode multiple steps adaptive predictive control strategy is brought forward, which can import more information about the system's dynamical characteristics. Experiments of constant water pressure equipment's control prove that this proposed algorithm can effectively alleviate system's oscillation when used to control a plant with severe nonlinearity, and that this algorithm shows good robustness for outer disturbances. So it is suitable to be generalized to the design of complex industrial process controller.
\end{abstract}

\section{Introduction}

Severe nonlinearities universally exist in Complex industrial processes, such as distillation process, $\mathrm{pH}$ neutralization process, etc. The control of severe nonlinearities is an urgent task in the field of process control all along ${ }^{[8]}$.

The standard predictive control algorithm based on OFS model[2,10] has many advantages such as non-sensibility of system structure and time-delay variances, low computational complexity, and robustness of parameter variances, etc., therefore, when dealing with linear or mildly nonlinearity, this algorithm can give satisfying control performance[2,3,11]. However, this standard algorithm is based on linear OFS model, so for severe nonlinear plant, this algorithm's performance is not good enough. Reference [1] gives an improved nonlinear control algorithm based on OFS model, but the adaptive effect is not satisfying when treating severe nonlinearity. Reference [1] combines Volterra Series with OFS model to identify nonlinear plant, and gains comparatively good control performance in the control simulation on CSTR (continuous stirring tank reactor) plant, but the computation load is too heavy, and real-time performance is not satisfying. Therefore, design an effective adaptive control algorithm for severe nonlinearities is an urgent task.

In this paper, firstly, the conception of Volterra Series is summarized. Then, the method to construct nonlinear hybrid model is introduced which based in OFS model combined with Volterra series. Based on this hybrid model, we propose an improved incremental mode nonlinear adaptive predictive control algorithm, which extend the 
control horizon from 1 step to 2 steps by using Gröbner basis function. The pump's output water pressure control experiment performances validate that this proposed algorithm can gain satisfying dynamic and steady-state performances. Moreover, compared with former nonlinear Laguerre predictive algorithm whose control horizon is one ${ }^{[1,6]}$, this one has better robustness under outside disturbances, besides, the vibrations of the control and controlled signals can be effectively decreased. Thus, the feasibility and superiority of this proposed algorithm are validated.

\section{Volterra Series of Nonlinear Systems}

Theorem $\mathbf{1}^{[9]}$ If the nonlinear system $F\left[u(t)_{-\infty}^{0}\right]$ fulfills the following 4 assumptions: 1)causal; 2)time-invariant; 3)input signal's power is finite; 4)the corresponding function of the system is continuous;

then, the system can be discomposed to the serial connection of a set of dynamic linear block and a memoryless nonlinear block.

Here, the linear block is $u(t)=\sum_{i=1}^{N} a_{i} P_{i}(t), a_{i}=\int_{-\infty}^{0} u(t) P_{i}(t) d t$, and $\left\{P_{i}(t)\right\}_{i=1}^{\infty}$ is set of complete orthonormal bases. In practice, we always choose the set of impulse response of this dynamic linear block $g_{i}(t)=P_{i}(-t)$, then

$$
a_{i}(t)=\int_{0}^{\infty} g_{i}(\tau) u(t-\tau) d \tau
$$

The memoryless nonlinear system is defined as

$$
F_{N}\left(a_{1}, \cdots, a_{N}\right) \stackrel{\Delta}{=} F\left[\sum_{i=1}^{N} a_{i} P(t)\right]
$$

\section{Theorem2 (Weierstrass Theorem) ${ }^{[5]}$}

Any continuous function $f(x)$ defined can be approximated uniformly to arbitrary accuracy on any finite, closed interval $[a, b]$ by polynomial of finite degree.

Definition1. If $F_{N}\left(a_{1}, \cdots, a_{N}\right)$ can be deployed to ${ }^{[2,11]}$

$$
F_{N}\left(a_{1}, \cdots, a_{N}\right)=\alpha+\left(\beta_{1} \alpha_{1}+\beta_{2} \alpha_{2}+\cdots+\beta_{N} \alpha_{N}\right)+\left(\gamma_{11} \alpha_{1}^{2}+\gamma_{12} \alpha_{1} \alpha_{2}+\cdots+\alpha_{N}^{2}\right)+\cdots
$$

then, define $h_{0}=\alpha$ as the 0 order Volterra kernel, $h_{1}(\tau)=\sum_{i=1}^{N} \beta_{i} g_{i}(\tau)$ as the 1 order Volterra kernel, $h_{2}\left(\tau_{1}, \tau_{2}\right)=\sum_{i, j=1}^{N} \gamma_{i j} g_{i}\left(\tau_{1}, \tau_{2}\right)$ as the 2 order kernel. 
So, from (3), we have

$$
y(t)=F[u(t)] \approx F_{N}\left(a_{1}, \cdots, a_{N}\right)=\sum_{n=0}^{\infty} y_{n}(t)
$$

here,

$$
y_{n}(t)=\int_{0}^{\infty} \int_{0}^{\infty} \cdots \int_{0}^{\infty} h_{n}\left(\tau_{1}, \tau_{2}, \cdots, \tau_{n}\right) \prod_{i=1}^{n} u\left(t-\tau_{i}\right) d \tau_{i}
$$

which is the Volterra series expression of nonlinear system for $u(t)$.

\section{Incremental Mode Nonlinear Adaptive Control Algorithm}

Theorem $3^{[12]}$ The incremental model linear control algorithm ${ }^{[12]}$ based on OFS model can eliminate steady-state error under output disturbances.

Theorem 3 has proved that the incremental OFS adaptive predictive control algorithm can trace set point curve without error, so it is superior to whole mode algorithm ${ }^{[2,6]}$. So, in order to enhance the closed-loop steady-state performance, we combine incremental mode OFS model with Volterra series, and construct a nonlinear adaptive algorithm based on this hybrid model to deal with server nonlinearities' control.

For convenience, we ignore the Volterra kernels whose orders are equal to or larger than 3, and use $N$ order Laguerre functional series, which is the most elegant one of the OFS family ${ }^{[10]}, \varphi_{i}(\tau), i=1, \cdots, N$, to approximate the 1 order and 2 order Volterra kernels which belong to the space $\mathbf{L}^{2}\left(R^{+}\right)$(square integrabel space on $[0, \infty)$, then

$$
\begin{gathered}
h_{1}\left(\tau_{1}\right)=\sum_{i=1}^{N} c_{i} \varphi_{i}\left(\tau_{1}\right) \\
h_{2}\left(\tau_{1}, \tau_{2}\right)=\sum_{n=1}^{N} \sum_{m=1}^{N} c_{n m} \varphi_{n}\left(\tau_{1}\right) \varphi_{n}\left(\tau_{2}\right)
\end{gathered}
$$

here, $c_{i}$ and $c_{n m}$ are all constant coefficients, so

$$
y(t)=h_{0}+\sum_{i=1}^{N} c_{i} l_{i}(t)+\sum_{n=1}^{N} \sum_{m=1}^{N} c_{n m} l_{n}(t) l_{m}(t)
$$

here,

$$
l_{j}(t)=\int_{0}^{\infty} \varphi_{j}(\tau) u(t-\tau) d \tau j=1, \cdots, N
$$

is the Laguerre functional series. 
Nonlinear Laguerre model's state space expression is stated as follows

$$
\begin{gathered}
L(k+1)=A L(k)+b u(k) \\
y_{m}(k)=c_{0}+C^{T} L(k)+L^{T}(k) D L(k)
\end{gathered}
$$

The definitions of $A, b$ can refer to reference [2]. $c_{0}=h_{0}, C^{T}=\left[c_{1}, \cdots, c_{N}\right]$, In the $k^{\text {th }}$ sampling period $L(k)=\left[l_{1}(k), \cdots, l_{N}(k)\right]^{T}$ is the Laguerre state vector, $u(k)$ is the input. $D=\left(c_{i j}\right)_{N \times N}(i=1, \cdots, N ; j=1, \cdots, N)$ $y_{m}(k)$ is the output of Laguerre model.

From (13) and extend the prediction time, we can get that

$$
y_{m}(k+i)=f(L(k+i))=c_{0}+C^{T} L(k+i)+L^{T}(k+i) D L(k+i)
$$

and

$$
L(k+i)=A^{i} L(k)+\bar{A}_{i} b u(k)
$$

Based on the reference [6], we have made the following 3 improvements.

1) import the output anti-disturbance amendment mechanism.

2) use RLS to update the coefficients matrices $C, D$ to implement adaptive mechanism.

3) use Gröbner based function algorithm to extend the control horizon from 1 to 2 , which can contain more information about the controlled plant's dynamic characteristics, and thus can yield better control performances. Its superiority will be validated in section 4 .

In detail, let

$$
y_{m}(k+i)=e_{0}(k, i)+e_{1}(k, i) u(k)+e_{2}(k, i) u^{2}(k)
$$

here

$$
\begin{gathered}
e_{0}(k, i)=c_{0}+C^{T} A^{i} L(k)+L^{T}(k)\left(A^{i}\right)^{T} D A^{i} L(k) \\
e_{1}(k, i)=C^{T} \bar{A}_{i} b+L^{T}(k)\left(A^{i}\right)^{T} D A_{i} b_{i}+b_{i}{ }^{T}\left(\bar{A}_{i}\right)^{T} D A^{i} L(k) \\
e_{2}(k, i)=b_{i}{ }^{T} \bar{A}_{i}{ }^{T} D \bar{A}_{i} b_{i} \\
\bar{A}_{i}=A^{i-1}+\cdots+A+I
\end{gathered}
$$

Let $Y_{m}(k+1)=\left[y_{m}(k+1), \cdots, y_{m}(k+P)\right]^{T}$, then we have 


$$
Y_{m}(k+1)=E_{0}+E_{1} u(k)+E_{2} u^{2}(k)
$$

here,

$$
E_{j}(k)=\left[e_{j}(k, 1), \cdots, e_{j}(k, p)\right]^{T}(j=0,1,2)
$$

Now import output amending mechanism and let

$$
Y_{P}(k+1)=Y_{m}(k+1)+[1, \cdots, 1]_{P \times 1}^{T}\left(y(k)-y_{m}(k)\right)
$$

and $Y_{r}(k+1)=\left[y_{r}(k+1), \cdots, y_{r}(k+P)\right]^{T}$, here $y_{r}(k+i)=\alpha^{i} y(k)+\left(1-\alpha^{i}\right) W(k)$, $\alpha$ is soften factor, $W(k)$ is set point. Let, $u(k)=u(k-1)+\Delta u(k)$.

The optimization control index is

$$
J=|| Y_{r}(k+1)-Y_{P}(k+1) \|^{2}+r \Delta u^{2}(k)
$$

Let $Y_{e}(k+1)=E_{0}(k)+E_{1}(k) u(k-1)+E_{2}(k) u^{2}(k-1)+[1, \cdots, 1]_{P \times 1}^{T}\left(y(k)-y_{m}(k)\right)$, $Y_{a}(k+1)=Y_{r}(k+1)-Y_{e}(k+1), E_{r}(k)=E_{1}+2 E_{2} u(k-1)$ then

$$
\frac{\partial J}{\partial \Delta u(k)}=s_{0}(k)+s_{1}(k) \Delta u(k)+s_{2}(k) \Delta u^{2}(k)+s_{3}(k) \Delta u^{3}
$$

$$
\begin{gathered}
s_{0}(k)=-E_{r}{ }^{T}(k) Y_{a}(k+1)-Y_{a}{ }^{T}(k+1) E_{r}(k) \\
s_{1}(k)=2\left[E_{r}^{T}(k) E_{r}(k)-E_{2}{ }^{T}(k) Y_{a}(k+1)-Y_{a}^{T}(k+1) E_{2}(k)+r\right] \\
s_{2}(k)=3\left[E_{r}{ }^{T}(k) E_{2}(k)+E_{2}{ }^{T}(k) E_{r}(k)\right] \\
s_{3}(k)=4 E_{2}{ }^{T}(k) E_{2}(k)
\end{gathered}
$$

Apply Newton method to solve the equation $\frac{\partial J}{\partial \Delta u(k)}=0$ (the Root function of MATLAB), and then can get control law $\Delta u(k)$ which minimizes $J$. Noted that the order of equation (21) is an odd number, equation (21) must have a real root at least.

From equations (10) and (13), we have that the output of the nonlinear Laguerre model is 


$$
y_{m}(k)=\theta^{T} \phi(k)
$$

here

$$
\begin{aligned}
& \theta^{T}=\left[c_{0}, \cdots, c_{N}, c_{11}, c_{12}, \cdots, c_{22}, \cdots, c_{N N}\right], \\
& \phi^{T}(k)=\left[1, l_{1}, \cdots, l_{N}, l_{1}^{2}, l_{1} l_{2}, \cdots, l_{2}^{2}, \cdots, l_{N}^{2}\right]
\end{aligned}
$$

This is a linear regression formulation, so $\theta^{T}$ can be identified by RLS online.

$$
\begin{aligned}
& P(k)=\frac{1}{\lambda}\left[I-\frac{P(k-1) \phi(k) \phi^{T}(k)}{\lambda+\phi^{T}(k) P(k-1) \phi(k)}\right] P(k-1) \\
& \hat{\theta}(k)=\hat{\theta}(k-1)+P(k) \phi(k)\left[y(k)-\phi^{T}(k) \hat{\theta}(k-1)\right]
\end{aligned}
$$

Now, we extend the control horizon from 1 to 2, then the equation (10) is improved into

$$
L(k+1)=\left[\begin{array}{cc}
A & \bar{A}_{1} B \\
A^{2} & \bar{A}_{2} B \\
\vdots & \vdots \\
A^{P-1} & \bar{A}_{P-1} B
\end{array}\right]\left[\begin{array}{ll}
L^{T}(k) & u(k-1)
\end{array}\right]^{T}+\left[\begin{array}{cc}
\bar{A}_{1} B & 0 \\
\bar{A}_{2} B & \bar{A}_{1} \\
\vdots & \vdots \\
\bar{A}_{P-1} B & \bar{A}_{P-2}
\end{array}\right] \Delta U(k)
$$

Accordingly, the optimization index (20) is changed into

$$
J=\left\|Y_{r}(k+1)-Y_{P}(k+1)\right\|^{2}+\|\Delta U(k)\|_{R}^{2}
$$

here, $\quad \Delta U(k)=[\Delta u(k), \Delta u(k+1)]^{T}, R=r I_{2 \times 2}(I$ is a unit matrix $)$ From $\frac{\partial J}{\partial \Delta U(k)}=0$, the equation (21) is changed into

$$
\begin{aligned}
& s_{m 30} \Delta u^{3}(k)+s_{m 20} \Delta u^{2}(k)+s_{m 10} \Delta u(k)+s_{m 000}+s_{m 21} \Delta u^{2}(k) \Delta u(k+1)+s_{m 01} \Delta u(k+1)+ \\
& s_{m 11} \Delta u(k) \Delta u(k+1)+s_{m 02} \Delta u^{2}(k+1)+s_{m 12} \Delta u(k) \Delta u^{2}(k+1)+s_{m 03} \Delta u^{3}(k+1)=0
\end{aligned}
$$

here, the polynomial equation coefficients $s_{m i j}(m=1,2 ; 0 \leq i \leq 3 ; 0 \leq j \leq 3)$ are determined by $Y_{r}(k+1), Y_{P}(k+1), L(k), u(k-1)$ and $R$.

Lemma $1^{[1,7]}$ Gröbner basis function can be used to solve the following function

$$
f_{1}(\Delta u(k), \Delta u(k+1))=f_{2}(\Delta u(k), \Delta u(k+1))
$$

here $f_{1}, f_{2}$ is the polynomial defined in the ring $Q^{2}(\Delta u(k), \Delta u(k+1))$. 
Lemma $2^{[1,7]}$ Assume $k\left[x_{1}, \cdots, x_{n}\right]$ is a polynomial ring, if $f_{1}, \cdots, f_{n} \in k\left[x_{1}, \cdots, x_{n}\right]$, then $\left\langle f_{1}, \cdots, f_{n}\right\rangle$ is an ideal of $k\left[x_{1}, \cdots, x_{n}\right]$, we defined it as the ideal caused by $f_{1}, \cdots, f_{n}$.

Theorem $4^{[1]}$ (Buchberger algorithm) let $\mathfrak{I}=\left\langle f_{1}, \cdots, f_{n}\right\rangle \neq\{0\}$ is a polynomial ideal, then a Gröbner basis of $\mathfrak{I}$ can be constructed in finite steps.

From Lemma1,2 and Theorem4, we can use Buchberger algorithm to calculate Gröbner basis, and then can solve the equation (32), the advantage of this algorithm is the ability to confirm the maximum order which can be reduced. Therefore, repeating Buchberger algorithm in this way, then, (32) can be rewritten as

$$
\begin{gathered}
v_{19} \Delta u^{9}(k)+\cdots+v_{11} \Delta u(k)+v_{10}=0 \\
\Delta u(k+1)=\frac{v_{20}(\Delta u(k))}{v_{21}(\Delta u(k))}
\end{gathered}
$$

here, the coefficients $v_{1 i}, 0 \leq i \leq 9$ are determined by $Y_{r}(k+1), Y_{P}(k+1), L(k)$, $u(k-1)$ and $R$, while $v_{20}, v_{21}$ are determined by by $Y_{r}(k+1), Y_{P}(k+1), L(k)$, $u(k-1), R$ and $\Delta u(k)$. Then we can use the root function of MATLAB online to compute the real root as the incremental model control law. The detailed deduction of (34), (35) can be referred to reference [7].

\section{Experiment Results}

As Fig.1 shown, this is an water output pressure control system, the executer is the Siemens MM440-typed converter, and the controlled plant is the Grundfos CHI-2 typed centrifugal pump (see the subfigure 2 of Fig.1). The signal flow is: the pressure sensor (see the subfigure 3 of Fig.1) transfers the output pressure into standard voltage signal (1-5V), and after A/D transmission, the signal is sent to PC serial port. The controller in PC calculates the control signal. After D/A transmission, the control signal is transferred into standard current signal (4-20mA), and implemented in the Siemens converter (see the subfigure 1 of Fig.1) to adjust the pumping frequency of Grundfos centrifugal pump.

In wide working area, the mechanism of this pump determined that it has severe nonlinearity. As fig. 2 shown, the relationships of the output pressure and the output water current under different rotating frequencies show server nonlinearity. So this pressure control system is a severe nonlinear one. However, in stable working area, this system fulfills the 4 assumptions of theorem1, so it can be decomposed to a set of dynamic linear block and a memoryless nonlinear block. Consequently, we can use our proposed algorithm to solve this problem. 


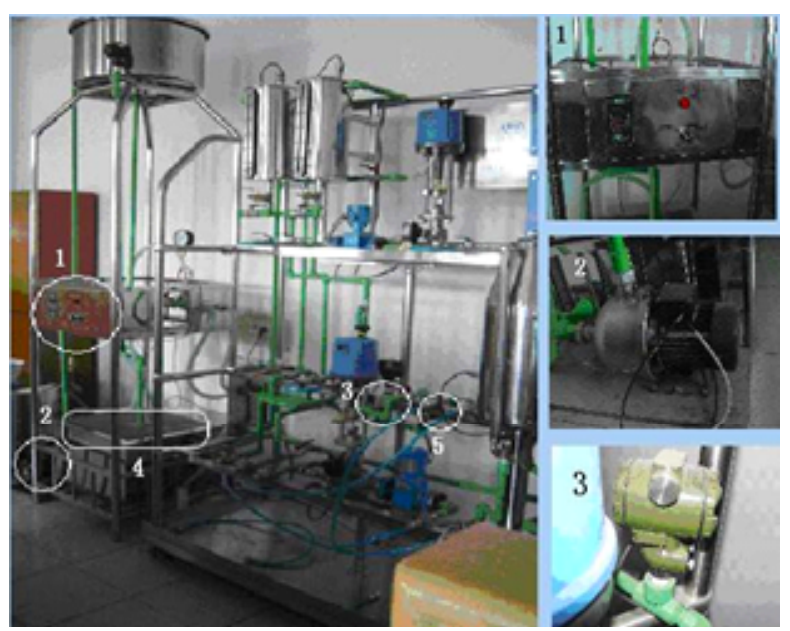

Fig. 1. Water pressure control system

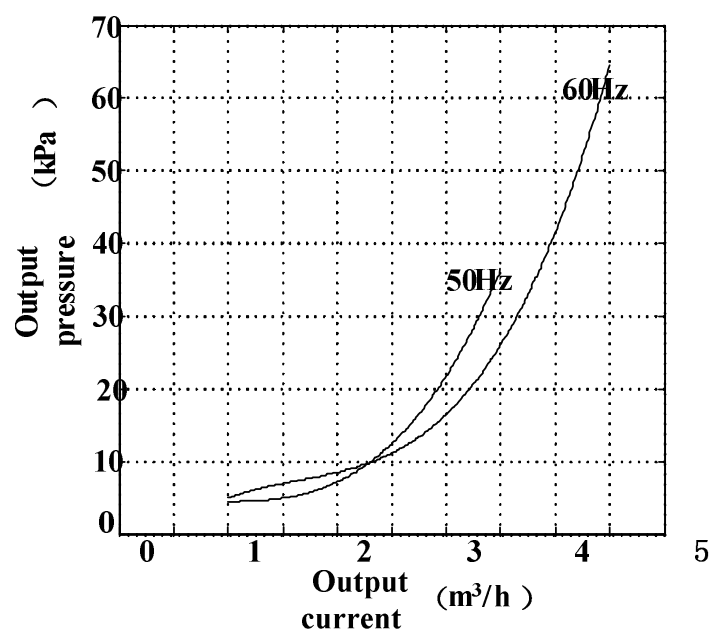

Fig. 2. Characteristic curves of Grundfos CHI-2 typed centrifugal pump

The control performances of the two nonlinear Laguerre predictive control algorithms whose control horizon is 1 (traditional algorithm) or 2(our improved algorithm) to trace $30.0 \mathrm{Kpa}$, 35.6 Kpa and 43.4 Kpa are shown in Fig.3 and Fig.4 respectively. In the $160^{\text {th }}$ sampling period (sampling period is 1 second), the opening of hand valve (see the subfigure 5 of Fig.1) is increased by $20 \%$, which imports an outside disturbance to test the robustness. The parameters of the traditional algorithm are: prediction horizon $P=6$, control horizon $M=1$, Laguerre series order $N=7$, time scaling factor $p=1.5$, forgetting factor $\lambda=0.7$, control weighting 
factor $r=0.9 ; \alpha=0.2$ the parameters of the improved algorithm are: $P=6, M=2, N=7, p=1.5, T=2, \lambda=0.2, \alpha=0.2, r=0.9$. From

Fig. 3 and Fig.4, we can conclude that these two algorithms both have certain robustness, and can eliminate steady-state error. The differences are that the improved algorithm can effectively decrease the vibrations of the control and controlled variables when compared with the traditional one. Besides, the overshooting is decreased remarkably under outside disturbance by our improved control algorithm. Thus, the control results validate the superiority of this proposed algorithm.
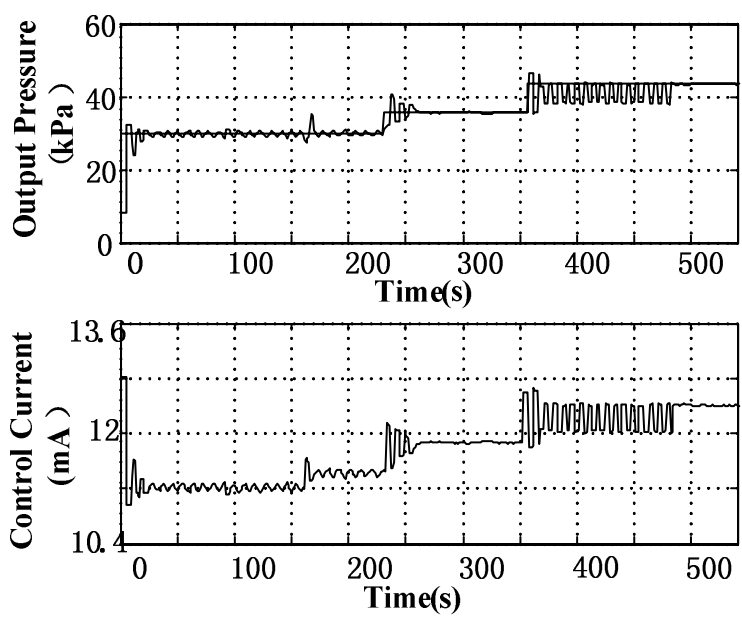

Fig. 3. Control performances of traditional control algorithm

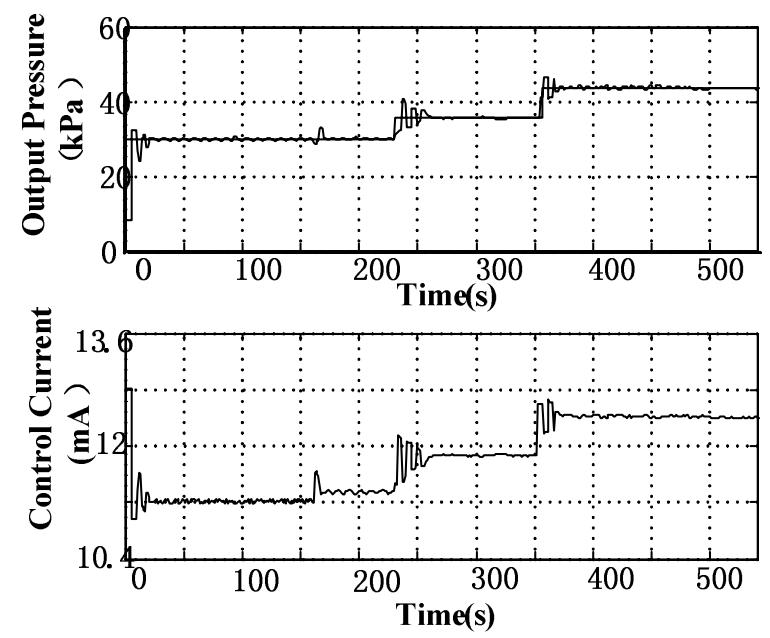

Fig. 4. Control performances of our improved control algorithm 


\section{Conclusion}

This paper extends the control horizon of the adaptive control algorithm based on OFSVolterra hybrid model from 1 to 2, and imports output anti-disturbance mechanism combined with online RLS OFS spectrum coefficients identification mechanism. In contrast to the traditional control algorithm whose control horizon is 1, this improved algorithm can effectively enhance the ability to solve server nonlinear plants, greatly decreases the vibrations and overshootings of the control and controlled variables. Besides, this algorithm also shows good robustness for outside disturbance, so is suitable for the design of complex industrial process controllers.

\section{Acknowledgement}

This paper is funded by Chinese National '985' High Level University Construction Fund (KY2701), and Hefei Key Scientific Research Project Fund (He Ke 2002-45).

\section{References}

1. Buchberger,B. Grobner bases: An algorithmic method in polynominal ideal theory. In N.K.Bose, editor, Multidimensional Systems Theory, D.Reidel Publishing Company, Dorderrecht, (1985) 184-232

2. Dumont,G.A., Zervos, C.C., et al.: Laguerre-based adaptive control of PH in bleach plant extraction stage. Automatica, 26(4), (1990) 781-787.

3. Li, S.F., Li,Y.Q., Xu. Z.F., Chen, Z.B.,: An extension to Laguerre model adaptive predictive control algorithm, Journal of China University of Science and Technology, 31(1), (2001) 92-98

4. Marmarelis V.Z., et al.: Volterra models and three-layer perceptrons, IEEE Transaction on neural networks, 8(6), (1997) 1421-1430

5. Michael, A.H., Dale, E.S.: Nonlinear Process Control, Prentice Hall PTR, New Jersey, (1997), 40-41

6. Parker, R.S., et al.: Nonlinear model predictive control of continuous bioreactor at near optimum conditions, Proceedings of American Control Conference, Philadelphia, Pennsylvania, (1998) 2549-2553

7. Parker, R.S., Nonlinear model predictive control of a continuous bioreactor using approximate data-driven models. Proceedings of automatic control conference. Anchorage, AK May 8-10, (2002) 2885-2890

8. Sbarbaro,D., Bassi,D.: A nonlinear controller based on self-organizing maps, IEEE,(1995) 1774-1777

9. Seretis, C, et al.: Nonlinear dynamical system identification using reduced Volterra models with generalized orthonormal basis functions. Proceedings of American Control Conference, Albuquerque, New Mexico, June (1997) 3042-3046

10. Wang, L.P.: Discrete model predictive controller design using Laguerre functions, Journal of Process Control, 14(2), (2004) 131-142

11. Zhang, H.T., Xu, Z.F., Li, S.F.: An adaptive predictive control based on Laguerre Function Model for diffusion furnace, System Engineering and Electronics 24(4),(2002) 54-57

12. Zhang, H.T., Chen Z.H., Qin, T.: An adaptive predictive control strategy for water recycling irrigation system of cropper, Journal of Information and Control of China 32(7), (2003) 586-590 